Please do not remove this page

RMIT

UNIVERSITY

\title{
Strain response of stretchable micro-electrodes: Controlling sensitivity with serpentine designs and encapsulation
}

Gutruf, Philipp; Walia, Sumeet; Ali, Md Nur; Sriram, Sharath; Bhaskaran, Madhu

https://researchrepository.rmit.edu.au/esploro/outputs/9921859533001341/filesAndLinks?institution=61RMIT_INST\&index=null

Gutruf, P., Walia, S., Ali, M. N., Sriram, S., \& Bhaskaran, M. (2014). Strain response of stretchable micro-electrodes: Controlling sensitivity with serpentine designs and encapsulation. Applied Physics Letters, 104(2), $021908-1-021908$ - 4. https://doi.org/10.1063/1.4862264

Document Version: Accepted Manuscript

Published Version: https://doi.org/10.1063/1.4862264

Repository homepage: https://researchrepository.rmit.edu.au

(c) 2014 AIP Publishing LLC

Downloaded On 2023/04/27 01:18:52 +1000 
Thank you for downloading this document from the RMIT Research Repository.

The RMIT Research Repository is an open access database showcasing the research outputs of RMIT University researchers.

RMIT Research Repository: http://researchbank.rmit.edu.au/

\section{Citation:}

Gutruf, P, Walia, S, Ali, M, Sriram, S and Bhaskaran, M 2014, 'Strain response of stretchable micro-electrodes: Controlling sensitivity with serpentine designs and encapsulation', Applied Physics Letters, vol. 104, no. 2, pp. 021908 -1-021908 - 4.

See this record in the RMIT Research Repository at:

http://researchbank.rmit.edu.au/view/rmit:23018

Version: Accepted Manuscript

Copyright Statement: (c) 2014 AIP Publishing LLC

Link to Published Version:

http://dx.doi.org/10.1063/1.4862264 


\title{
Strain response of stretchable micro-electrodes: Controlling sensitivity with serpentine designs and encapsulation
}

\author{
Philipp Gutruf, Sumeet Walia, Md Nur Ali, Sharath Sriram, ${ }^{\text {a) }}$ and Madhu Bhaskaran ${ }^{\text {a) }}$ \\ Functional Materials and Microsystems Research Group, School of Electrical and Computer Engineering, \\ RMIT University, GPO Box 2476, Melbourne, Victoria, Australia.
}

(Received

\begin{abstract}
The functionality of flexible electronics relies on stable performance of thin film micro-electrodes. This Letter investigates the behavior of gold thin films on polyimide, a prevalent combination in flexible devices. The dynamic behavior of gold micro-electrodes has been studied by subjecting them to stress while monitoring their resistance in situ. The shape of the electrodes was systematically varied to examine resistive strain sensitivity, while an additional encapsulation was applied to characterize multilayer behavior. The realized designs show remarkable tolerance to repetitive strain, demonstrating that curvature and encapsulation are excellent approaches for minimizing resistive strain sensitivity to enable durable flexible electronics.
\end{abstract}

\section{KEYWORDS}

flexible devices, polyimide, gold, serpentine, encapsulation, strain 
Recently, flexible electronics have garnered increasing attention, due to the possible impact on mainstream electronics such as the development of highly sophisticated flexible mobile phones and wearable computing devices. ${ }^{1,2}$ Flexible electronic gadgets such as flexible keyboards are already on the market and their demand has been significantly increasing. Most of these commercial devices incorporate polyimide as the flexible substrate in order to define circuit paths for surfacemounted devices which are the rigid functional elements. Consequently, these devices are not fully flexible. Recent research has demonstrated that flexible thin film transistors, ${ }^{3}$ flexible solar cells, ${ }^{4}$ flexible batteries, ${ }^{5}$ and LCD displays ${ }^{6}$ can be realized on fully flexible substrates. However, in order to enable the development of next generation, truly flexible devices, a detailed analysis of every individual component of such flexible electronic devices has to be carried out.

Contrary to conventional electronic devices, where the technology was almost exclusively developed on silicon substrates due to its crystal structure, dupability and band gap, the choice of substrate in flexible electronics is not trivial due to the availability of many possible candidates such as polyethylene terephthalate $(\mathrm{PET})^{7}$ or polydimethylsiloxane (PDMS), ${ }^{8,9}$ each with its unique capabilities and disadvantages. Amongst them, polyimide provides very good adhesion, thereby enabling the metal films on top to stretch with the substrate until rupture, while still maintaining their electrical conductivity. ${ }^{10}$ This is in contrast to other flexible materials that suffer from temperature instability and incompatibility with acids or solvents, both crucial factors for microfabrication. Furthermore, polyimide is stable at relatively high temperatures of up to $400{ }^{\circ} \mathrm{C}$ and is unaffected by acid exposure, making it an excellent choice for a robust substrate that is compatible with current micro-fabrication techniques.

However, in order to fully realize truly flexible next generation electronic devices, featuring full functionality even under stress, a fundamental understanding of metalized polymer films and their behavior under load is extremely important. Furthermore, to ensure that a working device is 
not affected by the movement or deformation of the substrate, the resistive strain sensitivity must be reduced drastically.

In this work, we explore the design of electrodes that show minimal alteration in resistance when being subjected to strain. In exploring possibilities for strain-independent micro-electrode configurations, we chose a prevalent material combination of gold as the conductor and polyimide as the flexible substrate for experiments. Gold, as a conductor in flexible and stretchable devices, has been utilized and studied extensively. ${ }^{10-15}$ The research has shown that gold can be stretched dramatically and still maintain its electrical properties. The conducting mechanism of well-adhered gold thin films on polymeric substrates that are stretched far beyond the elastic limit of the metal, relies on strain delocalization, which enables the build-up of micro-island structures. Such islands stay in contact even at stress levels exceeding the bulk gold rupture strain. ${ }^{16}$ Furthermore, gold provides excellent conductivity and is inert at the elevated temperatures used in this experiment.

In order to study the strain sensitivity of gold-coated polyimide films, we use well-defined electrodes that are subjected to constant load and release. During the load-release cycling of the substrates, the resistance of these patterns is constantly measured in situ. To study resistive strain sensitivity, curved electrode patterns as well as encapsulation ${ }^{17}$ of the electrodes are used.

The gold micro-electrodes are realized on polyimide substrates utilizing standard microfabrication techniques. Initially, as a support substrate, a microscope glass slide is utilized [Fig. 1(a)], which is thoroughly cleaned to avoid contamination. The polyimide substrate (Kapton film, DuPont), with a thickness of $25.4 \mu \mathrm{m}$, is affixed by laminating it to the glass slide using a UV sensitive tape [Fig. 1(b)]. Gold films of $200 \mathrm{~nm}$ thickness are subsequently deposited on to the polyimide layers by electron beam evaporation (PVD75, Kurt J. Lesker) at a rate of $0.1 \mathrm{~nm} / \mathrm{s}$ [Fig. 1(c)]. The structuring of the gold layer is carried out using a standard photolithographic 
process followed by an aqua regia $\left(\mathrm{HCl}: \mathrm{HNO}_{3}: \mathrm{H}_{2} \mathrm{O}:: 3: 1: 2\right)$ etch process and a subsequent deionized water rinse to shape the gold layers [Fig. 1(d)]. The photoresist is then stripped with acetone, rinsed with isopropanol and dried with high purity nitrogen, to prepare the pattern for bonding. The subsequent interfacing of the electrodes is carefully carried out using the two-part silver bonding epoxy with gold ribbon. For the polyimide encapsulated samples, an identical process is carried out, albeit for an additional encapsulation step. To encapsulate the features, a positive photo-patternable polyimide (HD8820, HD Microsystems), was spin-coated on the pattern, followed by a $180 \mathrm{~s}$ soft-bake at $120^{\circ} \mathrm{C}$. The polyimide was then exposed to UV light for $45 \mathrm{~s}$ to expose the contact pads. The development is completed in a developer bath (AZ316 MIF, Microchem) for $2 \mathrm{~min}$. A schematic of the development result is illustrated [Fig. 1(f)]. The spun-on polyimide film is then cured in a furnace in a nitrogen environment. The curing process involves the samples being initially heated to $150{ }^{\circ} \mathrm{C}$ (with a ramp rate of $4{ }^{\circ} \mathrm{C} / \mathrm{min}$ ). This is followed by a slow ramp $\left(2.5^{\circ} \mathrm{C} / \mathrm{min}\right)$ to $320^{\circ} \mathrm{C}$, which is completed by a $60 \mathrm{~min}$ cure to fully cross-link the polyimide precursor. The curing process of the polyimide is completed with a gradual cooling to room temperature. The electrodes are then interfaced by bonding with conductive silver epoxy similar to the unencapsulated electrodes. Both unencapsulated and encapsulated samples [Fig. 1(e) and Fig. $1(\mathrm{~g})$, respectively] were then released from the glass substrate by a flood exposure of the adhesive UV sensitive tape.

A static reference sample was also produced analog on a glass slide on which a $200 \mathrm{~nm}$ thick layer of gold was deposited using electron beam evaporation. While a thin adhesion layer of titanium or chromium are normally required to promote adhesion of gold to glass, to enable directly comparable results to the polyimide samples, this layer was avoided. The metallization was carried out on the same hardware to ensure comparability. A standard lift-off procedure was carried out, producing an exact replica of the features on the polyimide substrate using the image reversal capabilities of the AZ5214E photoresist. 
The as-synthesized dog-bone shaped patterns (straight electrodes) shown in Fig. 2, were $2 \mathrm{~mm}$ in length, $100 \mu \mathrm{m}$ in width and terminated by $500 \mu \mathrm{m} \times 500 \mu \mathrm{m}$ bonding pads. In addition to the straight electrodes, serpentine electrodes with multiple $60^{\circ}, 90^{\circ}$, and $120^{\circ}$ curves were also realized in order to study the impact of curvature on the resistive strain sensitivity. The total length of these electrodes was $2,094 \mu \mathrm{m}, 3,141 \mu \mathrm{m}$, and 4,188 $\mu \mathrm{m}$ respectively. A width of $100 \mu \mathrm{m}$ and thickness of $200 \mathrm{~nm}$ of the electrode was consistently maintained for all samples.

Before the start of the dynamic tests, static tests were conducted to determine the initial resistance of the structures as well as the quality of the gold layers. A calibrated 4-point probe measurement setup ${ }^{18}$ was used to determine the sheet resistance of the reference sample as well as the gold film on the polyimide substrate. Figure 3 shows the sheet resistance of gold on glass to be $0.199 \Omega / \mathrm{sq}$ and a corresponding resistivity $\rho_{\text {Au/Glass }}=3.98 \times 10^{-8} \Omega \mathrm{m}$. This value is in agreement with values reported in literature for thin gold films on glass. ${ }^{19}$ Interestingly, a lower sheet resistance $\left(0.178 \Omega / \mathrm{sq}\right.$ and a corresponding resistivity of $\left.\rho_{\text {Au/Polyimide }}=3.57 \times 10^{-8} \Omega \mathrm{m}\right)$ is measured for the gold film grown on the non-crystalline polyimide substrate. The resistance measurements for the micro-scale electrodes were obtained using an Agilent digital multimeter after taking into account the wire resistance of the measuring equipment. Figure 3 shows that the measured values for different curvatures of the resistor patterns are in close agreement to the theoretical calculations that were obtained using the resistivity values from 4-point probe measurements.

Dynamic measurements were conducted in order to test the resistive strain sensitivity of the polyimide-based samples. The tests were conducted using a custom-built fully-automated stretching apparatus with a step resolution of $2.5 \mu \mathrm{m}$. The displacement of the samples was controlled by a linear stage actuated with a micrometer screw driven by a stepper motor (Trinamic PD-110-42, Germany). The resistance was measured using an Agilent multimeter after every displacement step. 
The stepper motor and multimeter were controlled by a LabView program which applies the displacement mappings automatically. The resistance measurements were acquired with accurate synchronization with the displacement.

Strain-resistance measurements were performed on the straight and serpentine electrodes, both non-encapsulated and with the spin-on polyimide encapsulation. Figure 4(a) shows a comparison of a straight micro-electrode with a strain of $3 \%$ applied 20 times with successive recovery to $0 \%$ strain. A significant reduction in the strain sensitivity of these straight microelectrodes is observed after encapsulation. The trend is visible throughout all tests, as curvature and encapsulation are considered [Figs. 4(b-d)]. However, the reduction in strain sensitivity of the encapsulated counterparts becomes less severe with increasing curvature. Furthermore, a constant reduction of strain sensitivity is achieved by curvature. When comparing the conventional (straight) unencapsulated design with the strongly curved and encapsulated micro-electrodes, a reduction in resistive strain sensitivity of $\sim 1,100 \%$ was achieved.

The observed result from the straight electrode indicates that elastic deformation is not the only parameter that influences the metal layer during the multiple stretching cycles. This appears counterintuitive since the resistance returns to its initial value after every stretching cycle. The change in resistance of an ideal straight gold resistor with pure dimensional elastic change in shape can be estimated by: ${ }^{20}$

$$
R=R_{0} \frac{\left(1+\varepsilon_{l}\right)}{\left(1-v \varepsilon_{l}\right)^{2}}
$$

where, $R_{0}$ is the initial resistance, $v$ the Poisson's ratio of gold (0.44), and $\varepsilon_{1}$ the induced strain (3\% or 0.03 ). A theoretical estimation of resistance change for serpentine electrodes is intricate as the strain distribution is not uniform throughout the curved area, as demonstrated by Gonzalez et al. ${ }^{21}$ 
Using Equation (1), a 5.8\% increase in resistance is estimated for the straight electrode. However, the experimental findings indicate a change of $\sim 22 \%$. This large discrepancy can be due to the micro-cracking in the gold film as a result of the applied stress. Such micro-cracking is a known phenomenon in well-adhered metal thin films on elastomeric substrates stretched beyond their elastic limit. ${ }^{16}$ This causes the resistance to increase beyond the theoretically expected level. The micro-cracks allow the gold to deform beyond its bulk rupture stain and return to its initial resistance. The occurrence of micro-cracking on the straight electrodes was verified by electron microscopy. ${ }^{10}$ It was further demonstrated that micro-cracking decreases rapidly with an increasing degree of electrode curvature. ${ }^{10}$

Such behavior can be explained using observations made in previous work on different elastomeric substrates. In previous work, extensive simulation and in situ testing of serpentine electrodes have shown that such a configuration improves maximum stretch ability of these patterns on elastomeric substrates. ${ }^{21}$ In case of metal layers on polyimide, the limiting factor for reversible stretchability is not failure of the conductor itself, but the plastic deformation of the substrate. However when operated within the elastic limit of the polyimide substrate, the resistive strain sensitivity of gold electrodes on polyimide is drastically improved when formed in a serpentine shape. Encapsulation is expected to improve the strain distribution by further delocalizing the stress in the films. This is expected to result in less micro-cracking, thereby making the elastic deformation dominant. As expected, the stretching experiments on straight and serpentine shaped electrodes demonstrate that the resistance is significantly lower after encapsulation (Fig. 4). When comparing the initial strain sensitivity of the non-encapsulated electrode with the encapsulated $120^{\circ}$ serpentine pattern a substantial reduction $(\sim 1,100 \%)$ in resistive strain sensitivity achieved. Throughout all tests all patterns show no notable degradation when subjected to multiple stretching cycles, verified by optical and electron microscopy imaging. 
In summary, we have examined the properties of gold thin films and straight and serpentine micro-electrodes on polyimide substrates. The static thin films were characterized by 4-point probe measurements to determine the resistivity of the thin films. Gold micro-electrodes on polyimide with varying serpentine shape with systematic variation of curvature were realized to test the impact on resistive strain sensitivity. Furthermore, we encapsulated these micro-electrodes with spin-on polyimide to evaluate the behavior when subjected to strain. Curvature and encapsulation proved to be independently effective tools to reduce the sensitivity of these patterns when subtracted to strain, with the combination of the two factors reducing strain sensitivity by $\sim 1,100 \%$ (for the encapsulated $120^{\circ}$ serpentine structures over unencapsulated straight micro-electrodes). These findings highlight the significance of electrode geometry design for durable flexible electronics, and the potential to use the curvature and encapsulation to control strain sensitivity. 


\section{ACKNOWLEDGMENTS}

P.G. acknowledges an Australian Government Endeavour International Postgraduate Research Scholarship. S.S. and M.B. acknowledge Australian Post-Doctoral Fellowships from the Australian Research Council (ARC) through Discovery Projects DP110100262 and DP1092717, respectively. S.S. acknowledges partial support from ARC Discovery Project DP130100062 and a Victoria Fellowship. The authors acknowledge the facilities and technical assistance of the Australian Microscopy and Microanalysis Research Facility at the RMIT Microscopy and Microanalysis Facility at RMIT University.

\section{NOTES}

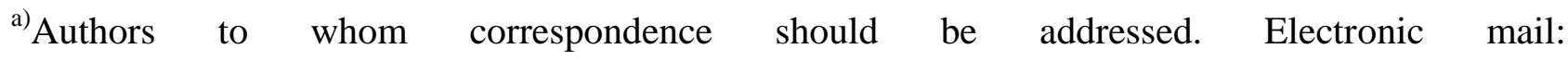
sharath.sriram@gmail.com, madhu.bhaskaran@gmail.com 


\section{REFERENCES}

${ }^{1}$ E. Huitema, G. Gelinck, B. van der Putten, E. Cantatore, E. van Veenendaal, L. Schrijnemakers, B.-H. Huisman, and D. de Leeuw, in Proceedings of 2003 IEEE International Solid-State Circuits Conference, (IEEE, 2003), p. 380.

2 J. Ross, "'Bendy' phones not such a stretch", The Australian, Sydney, 2013.

${ }^{3}$ W. B. Jackson, R. L. Hoffman, and G. S. Herman, Appl. Phys. Lett. 87, 193503 (2005).

${ }^{4}$ X. Mathew, J. P. Enriquez, A. Romeo, and A. N. Tiwari, Sol. Energy 77, 831 (2004).

${ }^{5}$ S. Xu, Y. Zhang, J. Cho, J. Lee, X. Huang, L. Jia, J. A. Fan, Y. Su, J. Su, and H. Zhang, Nature Comm. 4, 1543 (2013).

${ }^{6}$ P. Mach, S. J. Rodriguez, R. Nortrup, P. Wiltzius, and J. A. Rogers, Appl. Phys. Lett. 78, 3592 (2001).

${ }^{7}$ M. Zirkl, A. Haase, A. Fian, H. Schön, C. Sommer, G. Jakopic, G. Leising, B. Stadlober, I. Graz, and N. Gaar, Adv. Mater. 19, 2241 (2007).

${ }^{8}$ J. A. Rogers, T. Someya, and Y. Huang, Science 327, 1603 (2010).

${ }^{9}$ P. Gutruf, C. M. Shah, S. Walia, H. Nili, A. S. Zoolfakar, C. Karnutsch, K. Kalantar-zadeh, S. Sriram, and M. Bhaskaran, NPG Asia Mater. 5, e62 (2013).

${ }^{10}$ N. Lu, X. Wang, Z. Suo, and J. Vlassak, Appl. Phys. Lett. 91, 221909 (2007).

${ }^{11}$ B. M. Durnin, C. M. Shah, S. Sriram, and M. Bhaskaran, in Proceedings of Proceedings of SPIE, (SPIE, 2011), p. 820437.

${ }^{12}$ S. P. Lacour, J. Jones, Z. Suo, and S. Wagner, IEEE Electron Dev. Lett. 25, 179 (2004).

${ }^{13}$ S. Olliges, P. A. Gruber, S. Orso, V. Auzelyte, Y. Ekinci, H. H. Solak, and R. Spolenak, Scripta Mater. 58, 175 (2008).

${ }^{14}$ T. Adrega and S. P. Lacour, J. Micromech. Microeng. 20, 055025 (2010).

${ }^{15}$ S. Béfahy, S. Yunus, V. Burguet, J.-S. Heine, M. Troosters, and P. Bertrand, J. Adhes. 84, 231 (2008).

${ }^{16}$ S. P. Lacour, D. Chan, S. Wagner, T. Li, and Z. Suo, Appl. Phys. Lett. 88, 204103 (2006). 
${ }^{17}$ Y.-Y. Hsu, M. Gonzalez, F. Bossuyt, F. Axisa, J. Vanfleteren, and I. De Wolf, Thin Solid Films 519, 2225 (2011).

${ }^{18}$ F. M. Smits, Bell Syst. Technol. J. 37, 711 (1958).

${ }^{19}$ K. L. Chopra, L. C. Bobb, and M. H. Francombe, J. Appl. Phys. 34, 1699 (1963).

${ }^{20}$ P. Mandlik, S. P. Lacour, J. W. Li, S. Y. Chou, and S. Wagner, IEEE Electron Dev. Lett. 27, 650 (2006).

${ }^{21}$ M. Gonzalez, F. Axisa, M. V. Bulcke, D. Brosteaux, B. Vandevelde, and J. Vanfleteren, Microelectron. Reliab. 48, 825 (2008).

${ }^{22}$ See supplementary material at [URL to be inserted by AIP] for experimental details outlining the materials and methods used in this work. 


\section{FIGURE CAPTIONS}

FIG. 1. Schematic of the fabrication process for gold patterns on polyimide films with selective encapsulation. (a) A glass slide serves as a rigid support substrate during fabrication. (b) Polyimide film is temporarily affixed to glass by lamination. (c) A $200 \mathrm{~nm}$ thick gold layer is deposited on the polyimide surface by electron beam evaporation. (d) The gold micro-electrode patterns are defined by standard photolithography and wet etching. (e) The final freestanding device. (f) For further analysis, samples from (d) are encapsulated with spin-on polyimide, which is photolithographically defined with openings. (g) The final freestanding device with encapsulation.

FIG. 2. Schematic of the thin film metal patterns subjected to the static and dynamic tests. Straight resistors and serpentine resistors with arc segment angles of $60^{\circ}, 90^{\circ}$, and $120^{\circ}$ were studied.

FIG. 3. Static measurements of the reference gold pattern on glass and the pattern subject to test on polyimide vs. theoretical resistance of the pattern calculated based on resistivity from four-point probe measurements.

FIG. 4. In situ strain-resistance measurements comparing unencapsulated and spin-on polyimide encapsulated micro-electrodes with (a) straight geometry and (b)-(d) serpentine geometry with arc angles of $60^{\circ}, 90^{\circ}$, and $120^{\circ}$, respectively. 
(a)

(d)

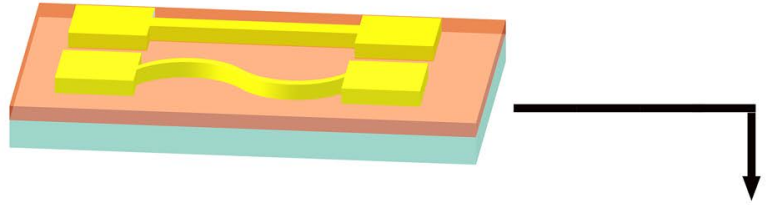

(b)

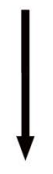

(f)

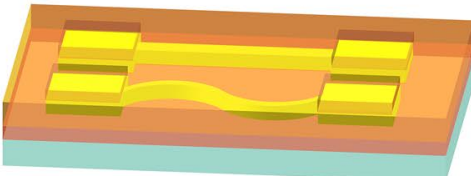

$\downarrow$

(c)

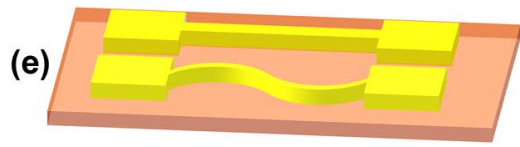

Unencapsulated
Glass

Polyimide (Film)
Gold

Polyimide (Spin-on) 


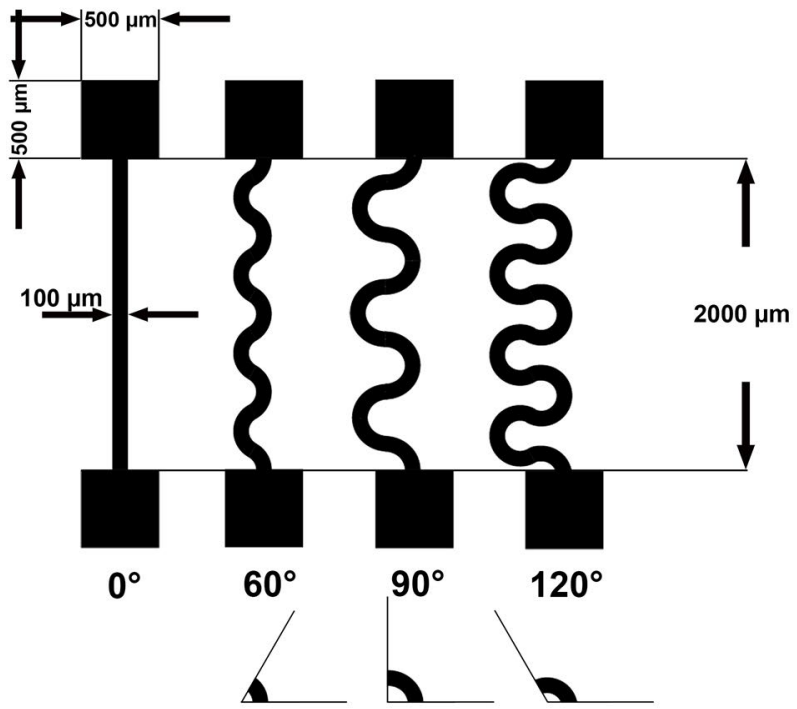




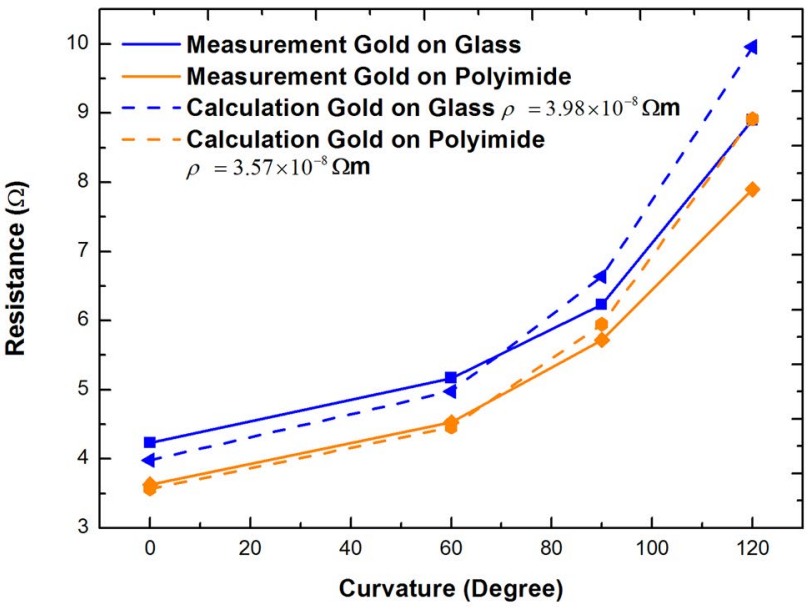




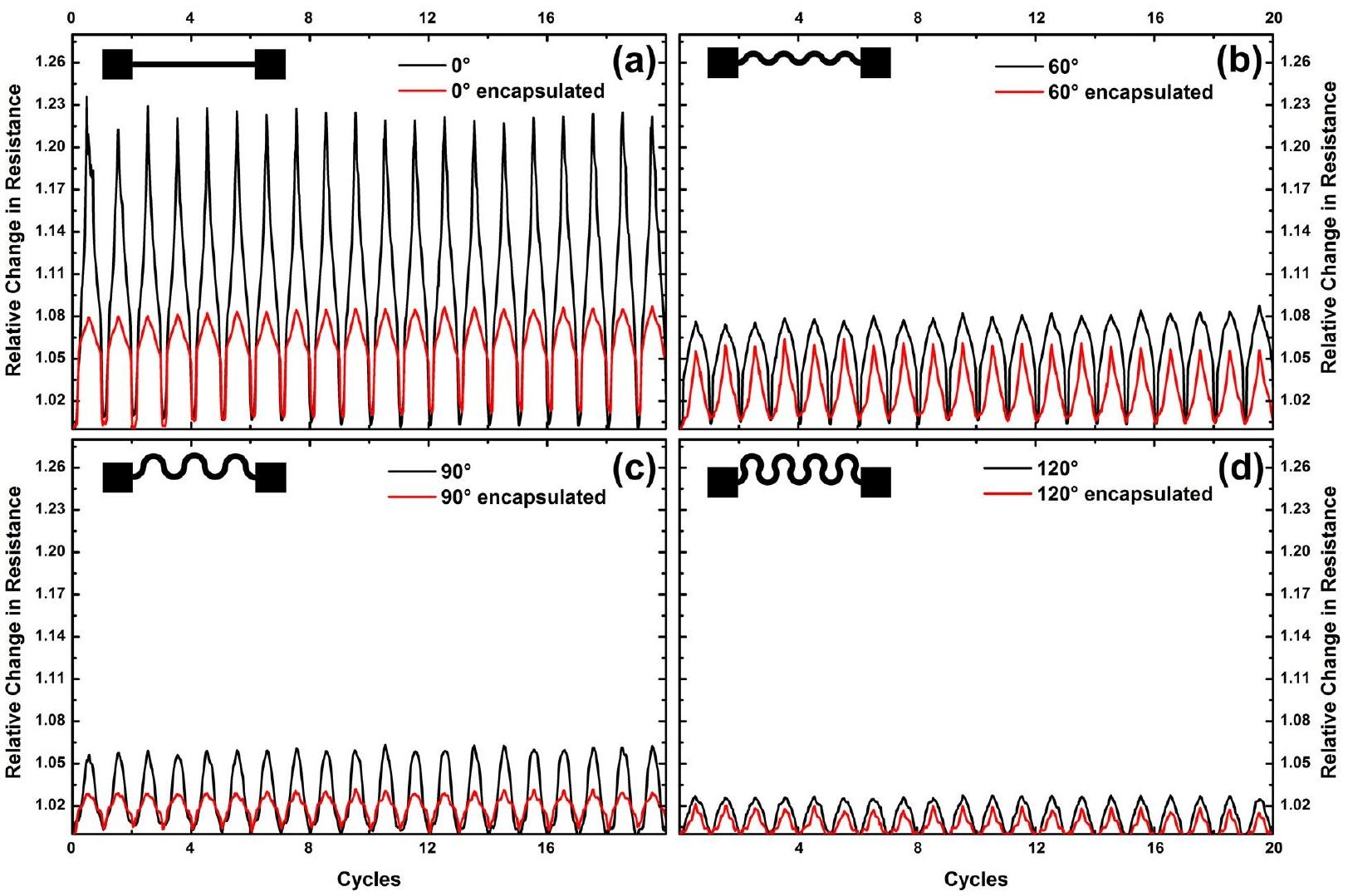

